\title{
Perfil epidemiológico de gestantes com HIV admitidas em uma maternidade de referência no Amapá
}

\author{
Epidemiological profile of pregnant women with HIV Admitted to a Non-Amapá Maternity \\ Unit
}

Perfil epidemiológico de mujeres embarazadas con VIH ingresadas en una maternidad de referencia en Amapá

Sara Pinto Teixeira ${ }^{1}$, Diana Silva de Aguiar ${ }^{1}$, Camila Rodrigues Barbosa Nemer ${ }^{1}$, Rubens Alex de Oliveira Menezes ${ }^{2 *}$.

\section{RESUMO}

Objetivo: Analisar o perfil epidemiológico de gestantes portadoras do HIV atendidas no Hospital da Mulher Mãe Luzia (HMML) na cidade de Macapá-AP no período de 2008 a 2018. Métodos: Trata-se de um estudo descritivo, de cunho documental e natureza quantitativa. O local de estudo é o HMML, para a obtenção dos dados, utilizou-se informações de documentos secundários, contidas nas fichas de notificação do Sistema de Informação de Agravos de Notificação (SINAN), que foram disponibilizadas pela Superintendência de Vigilância em Saúde (SVS). Resultados: No período de 2008 a 2018, foram registradas 253 notificações de gestantes com HIV no HMML, sendo que a maioria tinha idade entre 20 e 29 anos, era da cor parda (75,1\%), possuíam o ensino fundamental completo ou incompleto (39,5\%), era proveniente da capital Macapá, realizou o pré-natal $(81,8 \%)$ e teve seu diagnóstico durante o pré-natal $(40,7 \%)$. Conclusão: O conhecimento das características que permeiam este grupo específico pode subsidiar a elaboração de políticas e ações de combate ao HIV/AIDS em mulheres com o mesmo perfil e para o incremento na prevenção da transmissão vertical do vírus através da detecção precoce e adesão em tempo ao tratamento adequado.

Palavras-chave: HIV, Gravidez, Epidemiologia.

\begin{abstract}
Objective: To analyze the epidemiological profile of pregnant women with HIV treated at the Luzia Women's Mother Hospital (HMML) in the city of Macapá-AP from 2008 to 2018. Methods: This is a descriptive study of documentary cunho and quantitative nature. Or local study and HMML, for obtaining two dice, use of secondary documents, contained in the notifications of the Notification Information System (SINAN), which are made available by the Supervision of Surveillance in Saúde (SVS). Results: From 2008 to 2018, there were 253 reports of pregnant women with HIV in the HMML, most of them aged between 20 and 29 years old, mixed race $(75.1 \%)$, complete or incomplete elementary school. (39.5\%), coming from the capital of Macapá, performed prenatal care $(81.8 \%)$ and was diagnosed during prenatal care $(40.7 \%)$. Conclusion: The knowledge of the characteristics that permeate this A specific group can support the development of policies and actions to combat HIV / AIDS in women with the same profile and to increase the prevention of vertical transmission of the virus through early detection and timely adherence to appropriate treatment.
\end{abstract}

Key words: HIV, Pregnancy, Epidemiology.

1 Universidade Federal do Amapá (UNIFAP), Macapá-AP.

2 Laboratório de Estudos Morfofisiológicos e Parasitários da Universidade Federal do Amapá (UNIFAP). Macapá-AP. *E-mail: rubens.alex@unifap.br 


\section{RESUMEN}

Objetivo: Analizar el perfil epidemiológico de mujeres embarazadas con VIH tratadas en el Hospital Madre de Mujeres Luzia (HMML) en la ciudad de Macapá-AP de 2008 a 2018. Métodos: Este es un estudio descriptivo, de naturaleza documental y cuantitativa. El lugar de estudio es el HMML, para obtener datos, utilizamos información de documentos secundarios contenidos en los formularios de notificación del Sistema de Información de Enfermedades de Notificación (SINAN), que fueron puestos a disposición por la Superintendencia de Vigilancia Sanitaria (SVS). Resultados: De 2008 a 2018, hubo 253 informes de mujeres embarazadas con VIH en el HMML, la mayoría de ellas con edades entre 20 y 29 años, de raza mixta (75.1\%), escuela primaria completa o incompleta. (39.5\%), proveniente de la capital de Macapá, realizó atención prenatal $(81.8 \%)$ y fue diagnosticada durante la atención prenatal $(40.7 \%)$. Conclusíon: El conocimiento de las características que impregnan este grupo específico puede apoyar la elaboración de políticas y acciones para combatir el VIH / SIDA en mujeres con el mismo perfil y para aumentar la prevención de la transmisión vertical del virus a través de la detección temprana y la adherencia en las mujeres. tiempo para el tratamiento adecuado.

Palabras clave: VIH, Embarazo, Epidemiología.

\section{INTRODUÇÃO}

O vírus da imunodeficiência humana (HIV) é responsável por causar a síndrome da imunodeficiência adquirida (AIDS), que é uma disfunção no sistema imunológico de seu hospedeiro que corresponde a sintomas clínicos decorrentes de uma vulnerabilidade excessiva a infecções oportunistas (LIMA SKSS, et al., 2017).

O perfil epidemiológico das pessoas afetadas pelo HIV sofreu diversas mudanças ao longo do tempo, sendo que, à princípio, o grupo populacional que mais era afetado pela AIDS era composto por homossexuais e bissexuais masculinos, pessoas que faziam transfusão sanguínea em tratamento como os hemofílicos e os usuários de drogas injetáveis. Atualmente, está ocorrendo um fenômeno de heterossexualização e feminização da doença (NETO JFR, et al., 2010).

Nesse sentido, pode-se concordar que a disseminação do HIV tem crescido consideravelmente entre o público feminino e já é uma realidade à nível mundial. Esse quadro traz consigo outras dificuldades, as quais repercutem fortemente na saúde pública. Uma delas é o aumento do número de gestantes com o HIV (PAES ALV, et al., 2017).

Uma vez que a maioria das mulheres que convive com o vírus encontra-se em plena vida reprodutiva, decorre também uma maior probabilidade de aumento do número de crianças infectadas a partir da transmissão vertical (TV). Isso justifica o fato de que $90 \%$ dos menores de 13 anos com AIDS no país são infectados por meio da TV (PAES ALV, et al., 2017; RAMOS JIM, et al., 2013). Há de se comentar que a TV é o meio em que a mãe transmite a doença para o filho, podendo ocorrer durante a gestação, no parto ou através da amamentação, sendo a TV a via principal de disseminação da infecção entre a população infantil (FILGUEIRAS PL, et al., 2014).

Desde o ano de 2006 a infecção pelo HIV é um agravo de notificação compulsória no Brasil (DOMINGUES RMSM, et al., 2018). De acordo com o boletim epidemiológico HIV/AIDS do Ministério da Saúde (MS) de 2017, foram notificados 108.134 casos de gestantes infectadas pelo HIV no período de 2000 a 2017. Além disso, dentro desse grupo predominam mulheres com idade entre 20 e 24 anos e com baixa escolaridade, sendo que a maioria reside na região Sudeste. Estima-se que cerca de 17.200 gestantes são infectadas pelo HIV no Brasil, a cada ano (SILVA RAR, et al., 2013).

Há uma tendência de crescimento do número de gestantes infectadas pelo HIV em todas as regiões do país, até mesmo por conta de uma mais ampla detecção da doença, possibilitada por um maior preparo do sistema de saúde que atualmente conta com testes-rápidos de HIV disponibilizados pela Rede Cegonha. As 
regiões norte e nordeste foram as que apresentaram maior incremento na taxa de detecção da infecção em gestantes, já que ambas apresentavam a taxa de 1,2 em 2006, passando para 2,9 e 2,0 casos/mil nascidos vivos em 2016, respectivamente (BRASIL, 2017).

No Amapá, no período de 2000 a 2017 foram notificados 401 casos de gestantes com HIV. Além disso, esta capital que possui taxa de detecção de HIV em gestantes igual a 4,4 casos/mil nascidos vivos, e encontrase no rol das 08 unidades federativas com taxa de detecção superior à nacional que foi de 2,6 no ano de 2016 (BRASIL, 2017).

A disseminação do HIV apresenta peculiaridades dependendo do contexto social e do segmento populacional em questão. Nesse entender, é imprescindível conhecer as especificidades de cada grupo afetado para que então seja possível elaborar planos de enfrentamento adequados a cada cenário estudado (GUANABARA MAO, et al., 2014).

Em razão disso, este estudo se propõe a analisar o perfil epidemiológico de gestantes portadoras do HIV atendidas no Hospital da Mulher Mãe Luzia (HMML) na cidade de Macapá-AP no período de 2008 a 2018. A principal motivação para essa pesquisa é o fato de que até o presente momento não há estudos publicados que tragam dados relacionando HIV na gestação no estado do Amapá.

\section{MÉTODOS}

Trata-se de um estudo descritivo, de cunho documental e natureza quantitativa. O local de estudo é o Hospital da Mulher Mãe Luzia (HMML), que é um hospital de referência nas áreas de ginecologia e obstetrícia, localizado na cidade de Macapá-AP. Adicionalmente, essa maternidade atende aos 16 Municípios do estado do Amapá e de municípios do Pará, tais como: Afuá, Almerim, Anajás, Breves, Chaves, Gurupá, Portel, Porto de Moz e Prainha, com destaque para as comunidades ribeirinhas do norte da ilha do Marajó, conhecida como região do ABC Paraense (Afuá, Breves e Chaves) (AMAPÁ, 2019a) (Figura 1).

Figura 1 - Localização geográfica do município de Macapá, estado do Amapá e dos municípios do Pará atendidos no HMML.
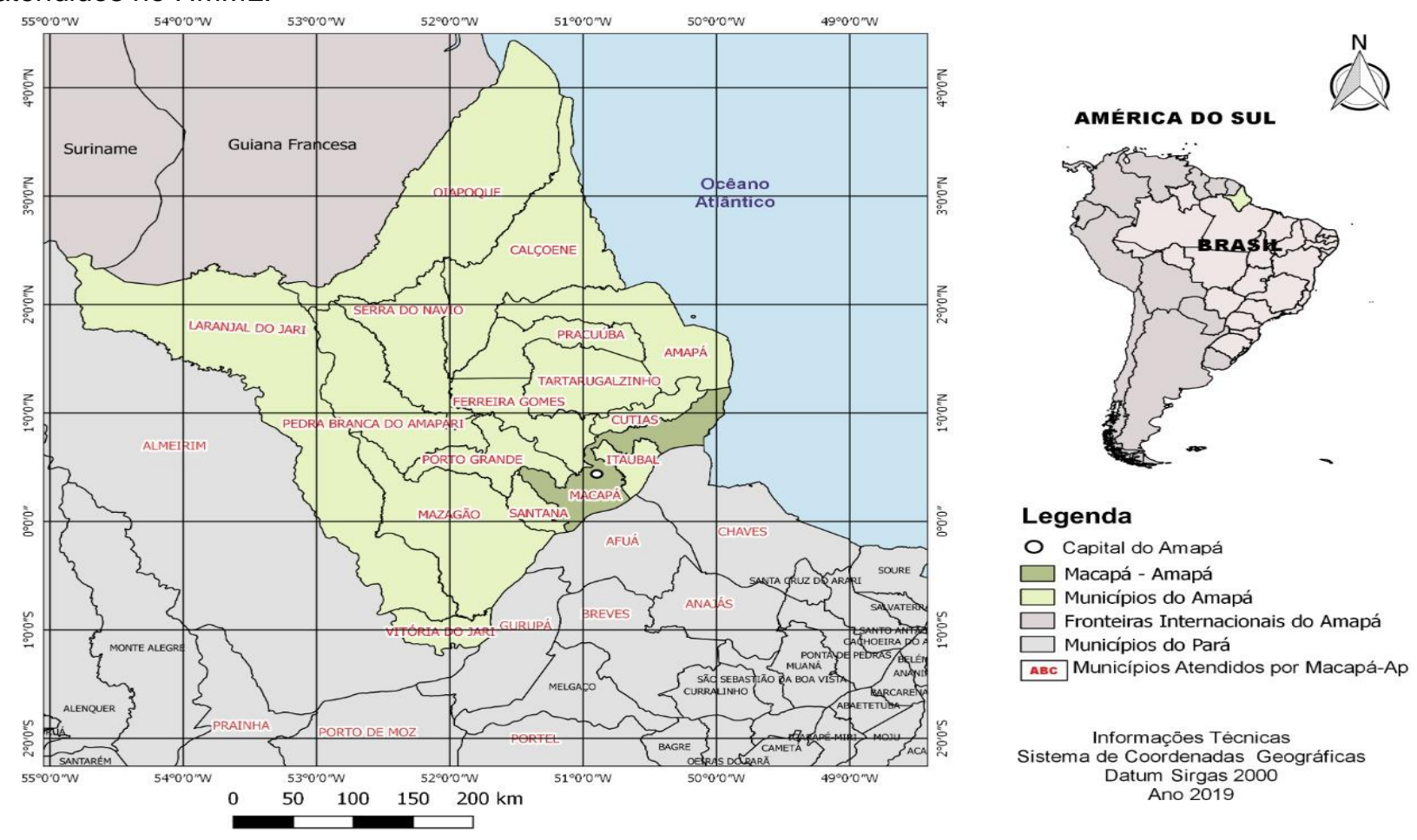

Fonte: Teixeira SP, et al., 2019. 
A população estudada foi composta por todas as gestantes portadoras de HIV, residentes do estado do Amapá, que foram notificadas no HMML, no período de 2008 a 2018. Para a obtenção dos dados, utilizou-se informações de documentos secundários, contidas nas fichas de notificação do SINAN, que foram disponibilizadas pela Superintendência de Vigilância em Saúde (SVS), órgão vinculado à Secretaria de Estado da Saúde do Amapá (SESA) (AMAPÁ, 2019b). A coleta de dados se deu no período de abril a junho de 2019, através de instrumento especifico construído pelos pesquisadores para este fim.

As informações coletadas foram organizadas de acordo com variáveis epidemiológicas preestabelecidas que são: sociodemográficas (idade, raça/cor, escolaridade e município de residência); de saúde (realização de pré-natal e momento da evidência laboratorial do HIV). Foi utilizado o software Excel para a realização dos cálculos da análise quantitativa e para organizar os dados em tabelas e gráficos. Esta pesquisa foi aprovada pelo Comitê de Ética em Pesquisa da Universidade Federal do Amapá através do parecer de número 3.171.715.

\section{RESULTADOS}

Os dados analisados demonstram que de 2008 a 2018, foram registradas 253 notificações de gestantes com HIV no HMML, de mulheres provenientes dos diversos municípios do Estado do Amapá, resultando em uma média de 23 notificações por ano. Além disso, conforme o (Gráfico 1), observou-se que os anos com maior número de notificações foram os de 2012 que contou com 12,3\% (31), 2014 com 13,4\% (34), 2015 com $11,9 \%$ (30) e 2016 com 15,4 \% (39), sendo este último o maior dentre todos. Adicionalmente, pode-se constatar que, a partir de 2011, houve um aumento progressivo no número de notificações até que a partir de 2016, houve uma considerável queda, levando à obtenção de apenas 10 casos em 2018.

Gráfico 1 - Distribuição do número de notificações do HMML de gestantes com HIV de 2008 a 2018.

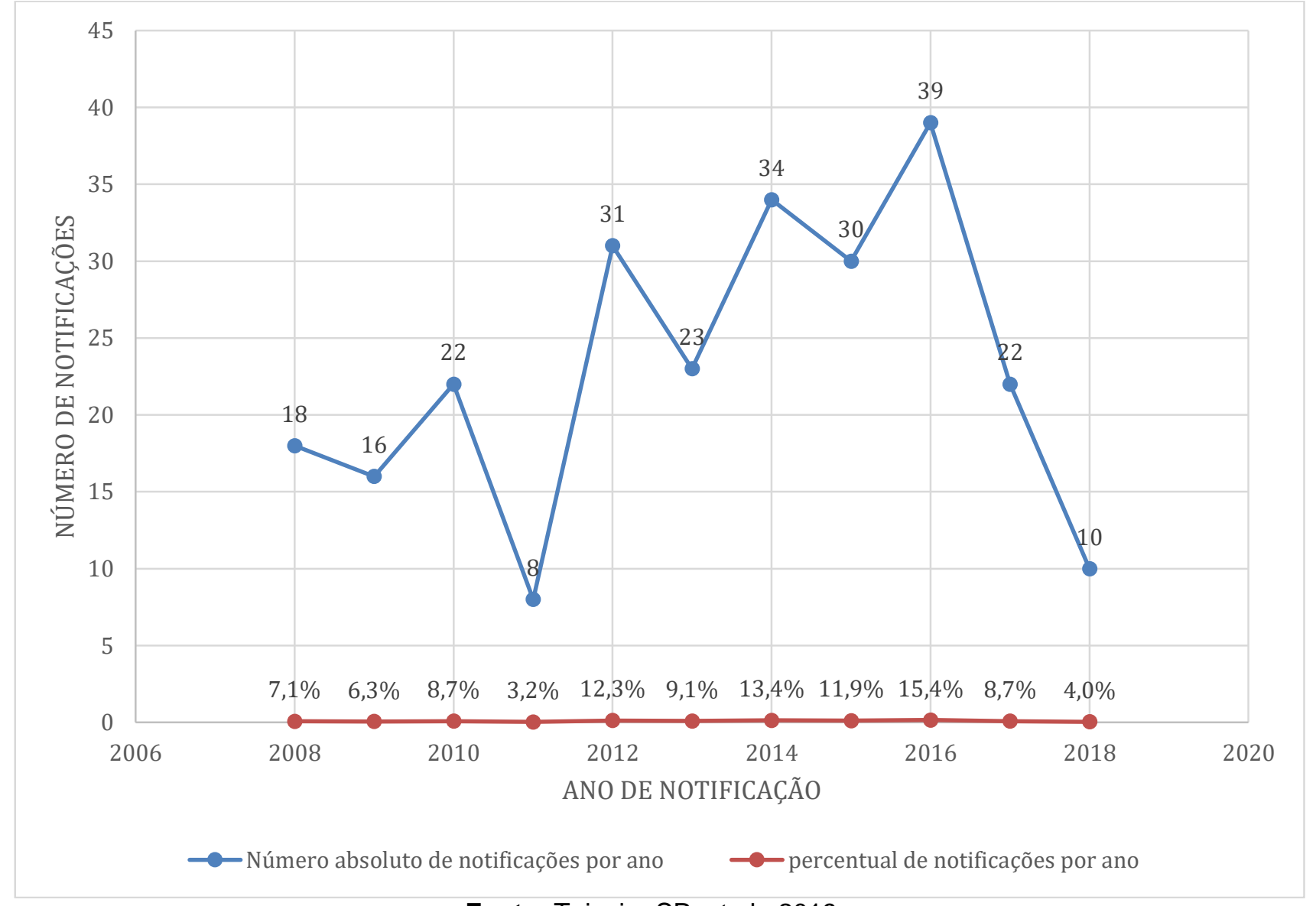

Fonte: Teixeira SP, et al., 2019. 
Os dados sociodemográficos deste estudo foram analisados através de 4 variáveis, quais sejam, idade, raça/cor, escolaridade e município de residência. Com relação à idade, a faixa-etária mais afetada foi de gestantes entre 20 e 29 anos em $52,2 \%$ dos casos (132), também observou-se que as faixa-etárias de 15 a 19 e 30 a 39 anos demonstraram números relevantes, com, respectivamente, 20,6\% (52) e 24,5\% (62) casos; as faixas de 10 a 14 e 40 a 49 contaram apenas com 0,4\% (1) e 2,4\% (6) dos casos.

A cor autodeclarada em $75,1 \%$ (190) dos registros das gestantes foi a parda. Sendo que $7,5 \%$ (19) eram brancas, $7,5 \%$ (19) pretas, indígenas 1,2\% (3) e amarelas $0,8 \%$ (2). No que concerne à escolaridade, 39,5\% (100) possuíam o ensino fundamental completo/incompleto, 36\% (91) ensino médio completo/incompleto, $6,7 \%$ (17) educação superior completa/incompleta e apenas $0,4 \%$ (1) era analfabeta.

Com relação ao município de residência, a maioria das gestantes, $82,2 \%$ (208), é proveniente da capital Macapá, cidade essa onde o hospital alvo deste estudo está localizado, e o segundo município com mais residentes foi Santana que contou com 5,9\% (15), o restante ficou distribuído entre os outros municípios do Estado do Amapá. Adicionalmente, a (Tabela 1), estão ilustradas às informações sobre os dados sociodemográficos supracitados.

Tabela 1 - Distribuição das gestantes com HIV segundo dados sociodemográficos.

\begin{tabular}{lcc}
\hline Variável & $(\mathrm{n})$ & $(\%)$ \\
\hline Faixa-etária & 0 & 0,0 \\
\hline Ignorado/branco & 1 & 0,4 \\
$10-14$ anos & 52 & 20,6 \\
$15-19$ anos & 132 & 52,2 \\
$20-29$ anos & 62 & 24,5 \\
$30-39$ anos & 6 & 2,4 \\
40-49 anos & & \\
\hline Raça/Cor & 20 & 7,9 \\
\hline Ign/Branco & 19 & 7,5 \\
Branca & 19 & 7,5 \\
Preta & 2 & 0,8 \\
Amarela & 190 & 75,1 \\
Parda & 3 & 1,2 \\
Indígena & & \\
\hline Escolaridade & 44 & 17,4 \\
\hline Ign/branco & 1 & 0,4 \\
Analfabeta & 100 & 39,5 \\
Ensino Fundamental completo/incompleto & 91 & 36 \\
Ensino médio completo/incompleto & 17 & 6,7 \\
Ensino superior completo/incompleto & & \\
\hline Município de Residência & 1 & 0,4 \\
\hline Amapá & 3 & 1,2 \\
Calçoene & 1 & 0,4 \\
Cutias & 2 & 0,8 \\
Ferreira Gomes & 1 & 0,4 \\
Itaubal & 1 & 0,4 \\
Laranjal do Jari & 208 & 82,2 \\
Macapá & 4 & 1,6 \\
Mazagão & 4 & 1,6 \\
Oiapoque & 3 & 1,2 \\
Pedra Branca do Amapari & 3 & 1,2 \\
Porto Grande & 15 & 5,9 \\
Santana & 2 & 0,8 \\
Serra do Navio & 2 & 0,8 \\
Tartarugalzinho & 3 & 1,2 \\
Vitória do Jari & 019 & \\
\hline & & \\
\hline
\end{tabular}

Fonte: Teixeira SP, et al., 2019. 
As variáveis de saúde investigadas neste estudo foram duas. A primeira é a realização do pré-natal (PN), a qual informa se o PN foi realizado ou não pelas gestantes, e a segunda é o momento da evidência laboratorial do HIV, a qual irá revelar se o diagnóstico da doença veio antes, durante ou após o PN, ou ainda, após o parto. De acordo com os dados coletados, $81,8 \%$ (207) das gestantes realizou o PN e, por outro lado, $12,6 \%$ (14) relataram não ter realizado (Grafico 2).

Gráfico 2 - Distribuição das gestantes com HIV segundo realização do PN.

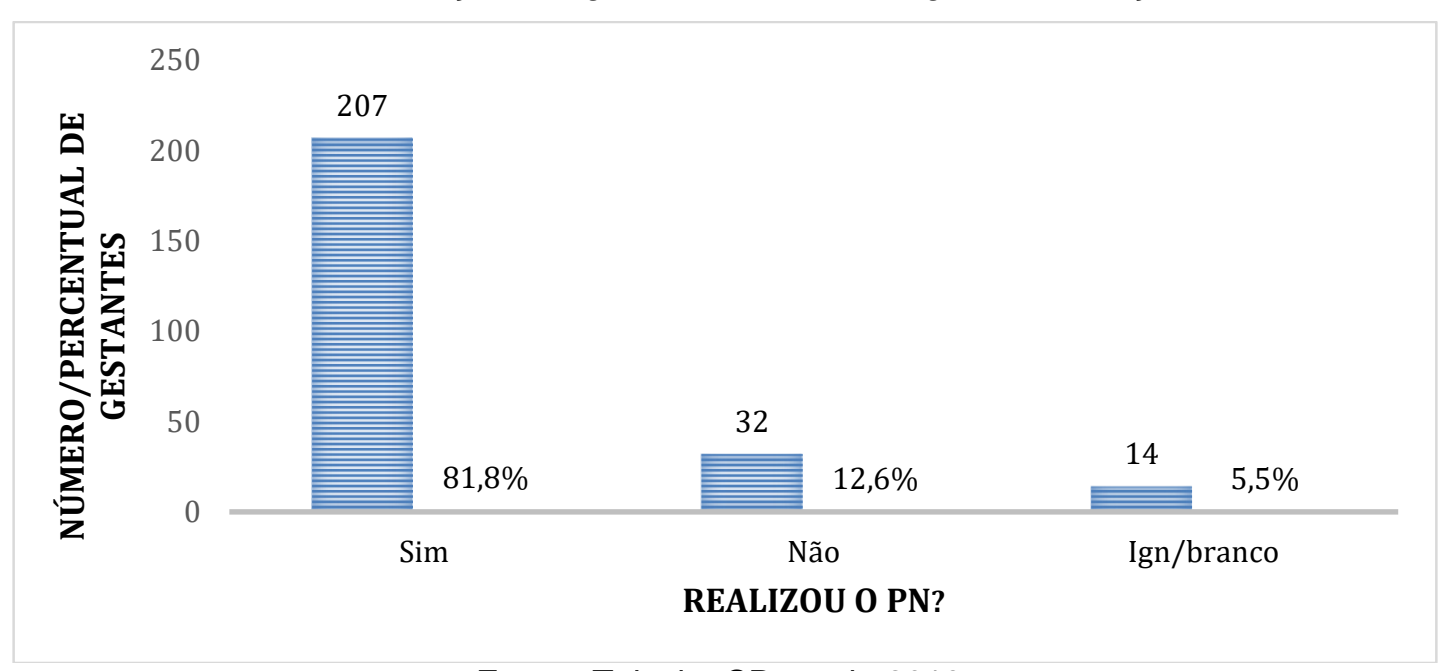

Fonte: Teixeira SP, et al., 2019.

No caso do momento da evidência laboratorial do HIV, a maioria soube do diagnóstico da doença durante o PN em $40,7 \%$ (103) dos casos, já 29,2\% (72) foi diagnosticada antes do PN, durante o parto foram $27,7 \%$ (70) e apenas 2,4\% (6) foi diagnosticada após o parto (Gráfico 3).

Gráfico 3 - Distribuição das gestantes com HIV conforme o momento da Evidência Laboratorial.

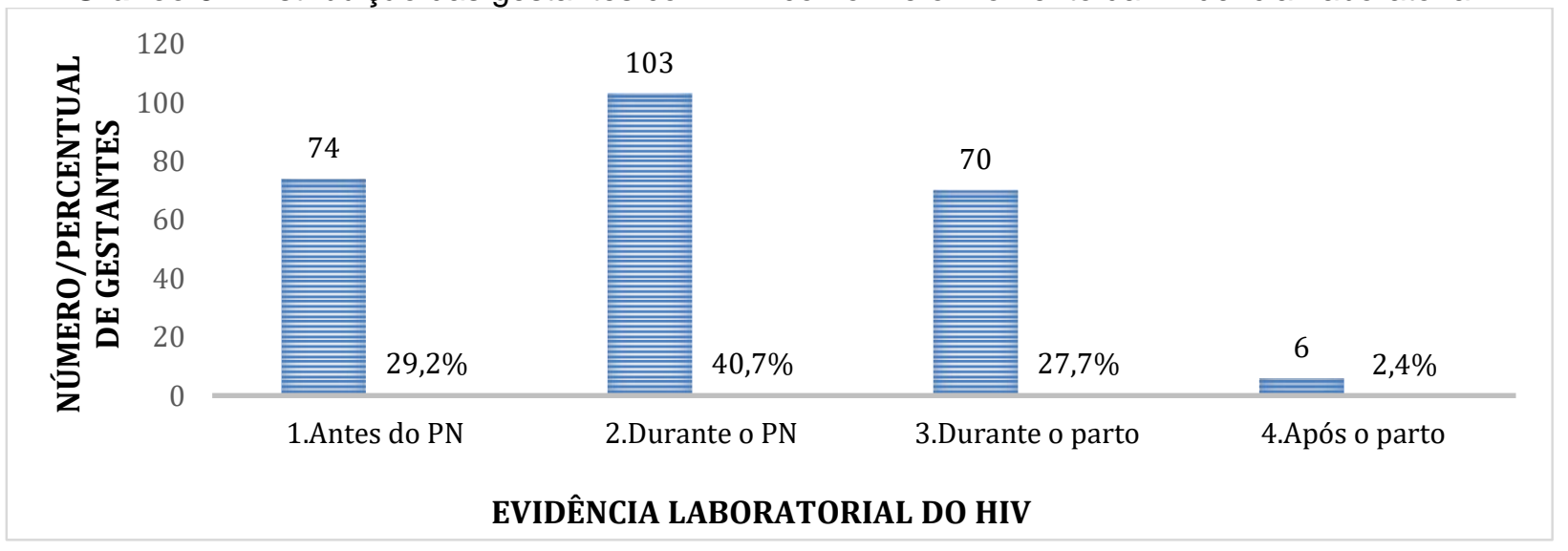

Fonte: Teixeira SP, et al., 2019.

\section{DISCUSSÃO}

O número de casos de HIV, embora ocorra no sexo masculino em maior número, nos últimos tempos vem apresentando um padrão de crescimento notadamente maior em mulheres, o que implica na atual "feminização" da doença. Existem situações específicas que corroboram com esse quadro, pois colocam a mulher em papel de maior susceptibilidade à essa infecção em relação aos homens, as quais vão desde razões biológicas (superfície da mucosa vaginal exposta é mais extensa e há maior concentração de HIV no sêmen do que no liquido vaginal) até questões socioculturais, tal realidade está relacionada ao aumento do risco de TV (NETO JFR, et al., 2010). 
A taxa de detecção do HIV em gestantes aumentou em $28 \%$ nos últimos 10 anos, ao passo que ocorreu um maior crescimento nas regiões norte e nordeste. Nesse sentido, a identificação do perfil epidemiológico do HIV em gestantes oferece informações preciosas para entender o contexto sócio espacial e os determinantes que estão relacionados ao aumento da vulnerabilidade dessas mulheres à essa infecção (LIMA SKSS, et al., 2017).

Neste estudo foram identificados 253 casos de gestantes com HIV admitidas no HMML de 2008 a 2018. Resultado semelhante foi observado na Paraíba, em que o número de notificações foi de 237, contudo, o período de investigação foi bem menor (2008-2012) (FILGUEIRAS PL, 2014). Já em outro estudo realizado em Alagoas, com período de investigação de (2007 a 2015), o número de gestantes notificadas foi bem maior comparado aos demais, com um total de 773 casos (SILVA CM, 2018). Adicionalmente, o ano em que mais ocorreram notificações no presente estudo foi o de $2016 \mathrm{com} 39$ casos, possivelmente por este ano, ter sido implementado sistemas com mais colaboradores aptos a notificar, bem como, maior treinamento e/ou qualificação de profissionais na realização e distribuição de testes rápidos em todo o país, sendo nesse período notificadas um total 7.823 casos de HIV em gestantes no Brasil (BRASIL, 2017).

Considerando as variáveis levantadas na tabela 1 , percebe-se que o principal padrão de mulheres afetadas ocorreu entre jovens, com baixo nível escolar. Pode-se observar que a faixa etária predominantemente acometida pelo HIV, durante a gestação, se deu entre 20 a 29 anos $(52,2 \%)$, com escolaridade igual ou inferior ao ensino fundamental (39,5\%). Notou-se também que uma considerável parcela das gestantes (36\%) possuía uma escolaridade que se enquadrava em ensino médio incompleto ou completo. Estes dados se assemelham com o estudo de Guanabara (2014), cujos resultados demonstraram esta mesma faixa-etária em $53,1 \%$ das gestantes, como também a escolaridade de $63,4 \%$ era de ensino fundamental completo ou incompleto e as que possuíam ensino médio completo ou incompleto representavam 32,6\%.

Estudos no Brasil demonstram que a maioria das gestantes infectadas com HIV têm idade entre 20 e 24 anos com escolaridade de $5^{\text {a }}$ a $8^{\text {a }}$ serie incompleta (BRASIL, 2017). Silva CM, et al. (2018) aponta a escolaridade como um importante indicador análogo às variáveis socioeconômicas, fazendo com que o aumento da doença em pessoas com menor grau de instrução seja um indicativo da irradiação da epidemia em camadas menos favorecidas da sociedade, fenômeno esse descrito como "pauperização".

Quanto à raça/cor da pele autodeclarada, há o predomínio de casos entre mulheres pardas $(75,1 \%)$, informação esta que pode ser justificada pelo fato de que no estado do Amapá $67,2 \%$ da população se autodeclara parda, segundo os dados do Instituto Brasileiro de Geografia e Estatística (IBGE) (2010). Similarmente, outros estudos como o que foi realizado no Pará, constatou que a maioria das gestantes com HIV era de cor parda. Até mesmo em regiões mais distantes, como o centro-oeste, foi detectado que a proporção de gestantes com HIV era de cor parda em 65,6\% dos casos (BARBOSA BLFA, et al., 2018; PAES ALV, et al., 2017). No Nordeste em Alagoas o estudo de Silva CM, et al. (2018), também ratifica esse resultado, com uma afirmativa de $72,1 \%$ de gestantes com HIV que se autodeclararam parda.

No Brasil, a maioria das gestantes portadoras de HIV (52,9\%) se autodeclara negra (sendo $11,4 \%$ pretos e $41,5 \%$ pardos) (BRASIL, 2018). A vulnerabilidade da população negra diante do HIV/aids, a invisibilidade e a violência contra mulher contribuem para o aumento de casos. A existência de desigualdade e a discriminação racial estão intimamente ligadas ao modo de operação de mecanismos sociais tais como: a educação escolar, a seletividade do mercado de trabalho, a pobreza e a organização familiar, provocando dessa forma, uma exclusão a população negra/parda, potencializando a marginalização de alguns grupos sociais, dessa forma, faz-se imprescindível um acolhimento e uma assistência focada para este grupo populacional com estratégias de promoção a saúde, no sentido da prevenção de doenças oportunistas (LÓPEZ LC, 2011). Para tanto, faz-se urgência definir as mulheres negras como população prioritária nas políticas públicas de IST/aids.O Estado do Amapá possui 16 municípios dentre eles a cidade de Macapá que corresponde a capital do estado, tem o número populacional segundo o último censo do IBGE de 669.526 pessoas (BRASIL, 2010). Durante o levantamento de dados em relação aos municípios de residência, observou-se, o predomínio de gestantes portadoras de HIV na referida capital, com o percentual de $82,2 \%$, sugere-se que esse grande quantitativo seja justamente pelo fato de que o hospital de referência seja localizado no município de Macapá. 
De forma parecida, no estudo de Guanabara MAO, et al. (2014) e Paes ALV, et al. (2017) a maioria das gestantes soropositivas estudadas residiam nas capitais Fortaleza e Belém, representando 65,3\% e 82,97\% do universo respectivamente. Já no estudo que ocorreu na cidade de Natal 51,06\% das gestantes HIV positivas eram de fora da capital, embora a pesquisa tenha sido realizada no hospital de referência de Natal. O processo de interiorização do HIV iniciou concomitante com a feminização, porém sua expansão ocorre diferentemente em cada região do país (CARVALHO CFS e SILVA RAR, 2014).

Com relação à variável realização do $\mathrm{PN}, 81,8 \%$ das gestantes realizou o $\mathrm{PN}$, conforme constata-se no gráfico 2. Em dois estudos realizados no nordeste brasileiro, foi constatado também que a maioria das gestantes teve acesso ao PN, com índices de 84,7\% e 89\%, respectivamente (FILGUEIRAS PL, et al., 2014; SILVA CM, et al., 2018). Embora estas pesquisas tragam resultados satisfatórios com relação à cobertura de $\mathrm{PN}$, a não realização deste ainda é uma realidade para muitas mulheres no país e pode estar relacionada à falta de conhecimento de sua própria condição e à deficiência dos serviços de saúde em realizar busca ativa dessas gestantes (SILVA CM, et al., 2018).

A evidência laboratorial do HIV demonstrou que o diagnóstico do HIV nas gestantes se deu principalmente durante o PN, em $40,7 \%$ dos casos, dado este semelhante aos resultados de um estudo de Alagoas, em que o diagnóstico no PN ocorreu para $41,3 \%$ das gestantes (SILVA CM, et al., 2018). Já em outras pesquisas realizadas no Sudeste e em Fortaleza, a maioria dos diagnósticos de Sorologia positiva para o HIV se deu anteriormente à gestação atual com representações percentuais de $42 \%$ e 57,1\%, respectivamente (BARBOSA BLFA, et al., 2018; GUANABARA MAO, et al., 2014).

Considerando que o PN é imprescindível no que tange ao diagnóstico precoce do HIV, o MS recomenda que sejam realizados testes rápidos para essa doença no primeiro e terceiro trimestres de gestação com vistas à diminuição do risco de transmissão materno-fetal. Em torno de $65 \%$ das ocorrências de TV se dá tardiamente na gestação, principalmente durante o trabalho de parto e parto propriamente dito, $35 \%$ ocorre intraútero, e há um risco de 7 a 22\% durante o aleitamento materno (BRASIL, 2012).

As próprias gestantes consideram a realização do PN fundamental, conforme constatou-se no estudo de Correio RSM, et al. (2015), pois, na percepção delas, este serviço é decisivo quando se fala em prevenção de doenças no período gestacional, sobretudo aquelas que podem ser transmitidas ao bebê. $E$ tudo isso porque, no PN é realizado o preparo físico e psicológico da mulher, além de atividades de educação em saúde que são importantes para a adoção de hábitos que irão promover a saúde do binômio mãe-bebê.

O conhecimento do diagnóstico do HIV para a maioria das gestantes se dá no período gestacional, contudo, as dificuldades de acesso de gestantes soropositivas ao PN são uma realidade presente e implicam em dificuldades na realização do diagnóstico precoce. Dentre os principais obstáculos, encontram-se questões socioeconômicas e demográficas que dificultam o acesso às consultas e atrapalham a realização do tratamento adequadamente. Além disso, o medo do preconceito e discriminação são fatores que podem impedir a gestante de procurar o serviço de saúde para a realização do teste anti-HIV. Dessa forma, é interessante que sejam elaboradas estratégias para captar essas gestantes para o PN e também para que se sintam acolhidas neste serviço, promovendo assim, a redução da TV (LIMA SS, et al., 2017).

Nesse sentido, cabe aqui ressaltar que o diagnóstico em tempo oportuno possibilita uma melhor análise do quadro clínico de cada gestante soropositiva, levando a um planejamento mais acertado da terapia antirretroviral (ARV) a ser utilizada, da via de parto mais adequada, à profilaxia ARV do recém-nascido (RN) e ao incentivo à não amamentação (BARBOSA BLFA, et al., 2018).

\section{CONCLUSÃO}

Neste estudo, o perfil epidemiológico das gestantes com HIV que foram admitidas no Hospital da Mulher Mãe Luzia na cidade de Macapá, no período de 2008 a 2018 foi caracterizado por gestantes jovens, de baixa escolaridade, residentes da capital Macapá, com predominância da cor parda, sendo que a maioria das gestantes realizaram o pré-natal e ressalta-se um número relevante que obteve seu diagnóstico durante este serviço. Entre as principais limitações desse trabalho há as informações classificadas como ignoradas ou em branco que se deram em número considerável dentre as variáveis, fazendo com que se perca informações 
relevantes sobre a população estudada e também demonstra que pode estar havendo uma falha no preenchimento das informações durante as notificações por parte dos profissionais da saúde.

\section{REFERÊNCIAS}

1. AMAPÁ. Secretaria de Estado da Saúde do Amapá. Hospital da Mulher Mãe Luzia: Serviço de Arquivo Médico e Estatística. Macapá: Secretaria de Estado da Saúde do Amapá, 2019a.

2. Secretaria de Estado da Saúde do Amapá. Superintendência de Vigilância em Saúde: sistema de informação de agravos de notificação. Macapá: Secretaria de Estado da Saúde do Amapá, 2019b.

3. BRASIL. Ministério da Saúde. Secretaria de Atenção à Saúde. Departamento de Atenção Básica. Atenção ao prénatal de baixo risco/Ministério da Saúde. Secretaria de Atenção à Saúde. Departamento de Atenção Básica. - Brasília: Editora do Ministério da Saúde, 2012.

4. Ministério da Saúde. Secretaria de vigilância e saúde. Boletim epidemiológico HIV/AIDS 2017. Ministério da saúde: 2017.

5. Ministério da Saúde. Secretaria de vigilância e saúde. Boletim epidemiológico HIV/AIDS 2018. Ministério da saúde: 2018.

6. BARBOSA BLFA, et al. gestantes hiv positivas e os fatores de risco relacionados à transmissão vertical do HIV. Revista de Enfermagem UFPE online, 2018; 12(1): 171-178.

7. CARVALHO CFS, SILVA RAR. Perfil sociodemográfico e de saúde de mulheres soropositivas em um pré-natal de alto risco. Revista Cogitare Enfermagem, 2014; 19(2):292-8.

8. CORREIO RSM, et al. Realização da sorologia para hiv no pré-natal: conhecimento e percepção da gestante. Revista enfermagem contemporânea, 2015, 4(1):12-22.

9. DOMINGUES RMSM, et al. Notificação da infecção pelo HIV em gestantes: estimativas a partir de um estudo nacional, Revista de Saúde Pública, 2018; 52(43): 1-9.

10. FILGUEIRAS PL, et al. Caracterização das gestantes portadoras de HIV no estado da paraíba, 2008 - 2012. Revista Brasileira de Ciências da Saúde, 2014; 18(2): 115-124.

11. GUANABARA MAO, et al. Gestantes com HIV/AIDS acompanhadas em serviços públicos. Revista de Enfermagem da UFPI, 2014; 3(2): 25-32.

12. INSTITUTO BRASILEIRO DE GEOGRAFIA E ESTATÍSTICA (IBGE). Censo Demográfico Brasileiro. Características da população e dos domicílios: Resultados do Universo. Amapá: IBGE, Brasília, 2010.

13. LIMA SKSS, et al. Caracterização das gestantes com hiv/aids admitidas em hospital de referência. Revista SANARE, $2017 ; 16(1): 45-51$.

14. LIMA, SS, et al. HIV na gestação: pré-natal, parto e puerpério. Revista ciência e saúde, 2017, 10(1):56-61.

15. LÓPEZ LC. Uma Análise das Políticas de Enfrentamento ao HIV/Aids na Perspectiva da Interseccionalidade de Raça e Gênero. Saúde e Sociedade, 2011; 20(3):590-603.

16. NETO JFR, et al. Perfil de adultos infectados pelo vírus da imunodeficiência humana (HIV) em ambulatório de referência em doenças sexualmente transmissíveis no norte de Minas Gerais. Revista Médica de Minas Gerais, 2010; 20(2): 22-29.

17. PAES ALV, et al. Perfil epidemiológico de gestantes com HIV acompanhadas em um serviço de assistência especializada em Belém-PA. Revista Interdisciplinar, 2017; 10(3): 100-109.

18. RAMOS JIM, et al. Perfil das Gestantes HIV/AIDS Admitidas em uma Maternidade de Alto Risco no Município de Aracaju/SE. Cadernos de Graduação- Ciências Biológicas e da Saúde, 2013; 1(17): 27-38.

19. SILVA CM, et al. Panorama epidemiologico do HIV/AIDS em gestantes de um estado do Nordeste brasileiro. Rev Bras Enferm, 2018; 71(supl1): 613-21.

20. SILVA RAR, et al. A Epidemia da AIDS No Brasil: Análise Do Perfil Atual. Revista de Enfermagem UFPE Online, 2013; 7(10): 6039-8. 